\title{
MODELAGEM UNI E BIVARIADA DA VARIABILIDADE ESPACIAL DE RENDIMENTO DE Pinus taeda L.
}

\author{
Itamar Antonio Bognola*, Paulo Justiniano Ribeiro Júnior**, Edson Antonio Alves da Silva***, \\ Christel Lingnau****, Antonio Rioyei Higa***** \\ *Eng. Agrônomo, Dr., Embrapa Florestas - iabog@cnpf.embrapa.br \\ **Eng. Agrônomo, Ph.D., Laboratório de Estatística e Geoinformação, UFPR - paulojus@ufpr.br \\ ***Físico, M.Sc., Depto. de Estatística, UNIOESTE - edsonsilva@unioeste.br \\ ****Eng ${ }^{\mathrm{a}}$. Florestal, Dr ${ }^{\mathrm{a}}$., Depto. de Ciências Florestais, UFPR - lingnau@ufpr.br \\ *****Eng. Florestal, Dr., Depto de Ciências Florestais, UFPR - higa@ufpr.br
}

Recebido para publicação: 20/03/2007 - Aceito para publicação: 11/10/2007

\begin{abstract}
O objetivo deste estudo foi avaliar a variabilidade espacial de rendimento de Pinus taeda L. em função de teores de argila do solo obtidos de um mapeamento detalhado de solos, na escala 1:10.000, em uma área localizada no município de Rio Negrinho, estado de Santa Catarina. Os dados dendrométricos para a realização deste estudo, coletados em parcelas de inventário florestal contínuo, foram obtidos de um povoamento com área de 2.252,11 ha. Propriedades do solo e rendimentos de Pinus taeda foram analisados através de métodos geoestatísticos, usando-se semivariogramas, modelos espaciais uni e bivariados e interpolação espacial por krigagem e cokrigagem para construção de mapas que descrevam o rendimento na região. Pelos semivariogramas, avaliou-se a estrutura de continuidade espacial do incremento médio anual volumétrico (IMA), pelo interesse prático nessa variável e também por ter sido a que melhor se correlacionou com os dados de solos. A comparação dos dois mapas obtidos de IMA obtidos pela modelagem uni e bivariada sugere que a última proporciona uma descrição mais detalhada do atributo de interesse na área, sendo o detalhamento da variabilidade espacial dependente do maior ou menor número de dados obtidos geograficamente para o estudo.
\end{abstract}

Resumo

Palavras-chave: Geoestatística; mapa de rendimento; levantamento de solo; krigagem e cokrigagem.

\begin{abstract}
Uni and bivariate modelling of the spatial variability of Pinus taeda $L$. The objective of this study was to assess spatial variability of Pinus taeda L. growth due to soil clay contents a function of the soil obtained from a detailed mapping, in the scale 1:10.000, in an area located in the municipality of Rio Negrinho, Santa Catarina State, Brazil. Data on forestry growth was collected from plots of continuous forest inventory, obtained from a population growing on an area of 2.252,11 ha. Soil properties and Pinus taeda crop yields were analyzed through geoestatistical methods using semivariograms and interpolation by kriging and cokriging in order to obtain maps describing the attribute of main interest over the study region. Semivariograms were used to evaluated the spatial structure of the dendrometric property annual average increment (IMA) which is of primary interest and has shown stronger correlation with soil attributes. The comparison of the maps obtained by the uni and bivariate models suggests the latter provides a more detailed description of the main variable over the area, being the resolution dependent on the availability of data obtained geographically for the study.

Keywords: Geoestatistic; yield map; soil survey; kriging and co-kriging.
\end{abstract}

\section{INTRODUÇÃO}

Avanços tecnológicos no segmento florestal têm mostrado a importância de se medir e obter aspectos da variação espacial e temporal de propriedades do solo que afetam o rendimento das espécies dos povoamentos florestais, com o objetivo de otimizar o gerenciamento do processo de produção. Cientistas da área de solos se preocupam com o problema desde o começo do século passado, mas somente nas décadas de 50 e 60 , com o avanço na teoria estatística espacializada, é que os cientistas 
começaram a perceber a potencialidade dessa metodologia para o manuseio de dados quantitativos, facilitando a sua compreensão (BURROUGH et al., 1994).

O desejável para todo empreendimento florestal é obter informações precisas e a baixo custo sobre a produção florestal. Um dos caminhos possíveis é o uso de um método que explore adequadamente as relações regionais existentes entre as unidades amostrais. Os métodos tradicionais usados para análise de inventários florestais utilizam uma medida de tendência central (média) e uma de dispersão (variância) para descrever um determinado fenômeno, sem levar em consideração as possíveis correlações entre as observações vizinhas (MELLO, 2004). Portanto, eles não exploram suficientemente as relações que possam existir entre as unidades amostrais. Já os métodos geoestatísticos podem avaliar melhor as estruturas de dependência espacial entre as características dendrométricas de uma espécie e as variáveis do seu meio físico, ou seja, obter resultados que sejam capazes de explorar adequadamente as relações espaciais existentes entre os dados dendrométricos e o meio abiótico. Isso é de fundamental importância para o inventário, o manejo e o planejamento florestal.

Vários trabalhos demonstram que as propriedades do solo são espacialmente dependentes (KRIGE, 1966; MATHERON, 1971; VIEIRA et al., 1983; TRANGMAR et al., 1985), ou seja, num certo domínio, a variância da diferença dos valores de determinada propriedade pode ser expressa como uma função da distância de separação dos pontos mensurados. Portanto, quando uma propriedade do solo varia de um local para outro, com algum grau de continuidade expresso pela dependência espacial, a metodologia geoestatística permite uma descrição espacial útil ao planejamento e ao controle das informações de produção da espécie florestal de interesse - no presente caso, P. taeda Linnaeus.

A utilização combinada de sistemas de informações geográficas (SIG) e métodos geoestatísticos permite amparar tecnicamente decisões estratégicas e complexas em relação ao sistema de manejo adotado, seus efeitos ambientais e a produtividade das diferentes essências florestais, contribuindo para o desenvolvimento de uma produção florestal sustentável. Dentro desse contexto, o objetivo deste estudo foi avaliar a variabilidade espacial do rendimento produtivo de $P$. taeda em função de propriedades do solo, por meio de técnicas geoestatísticas, considerando abordagens uni e bivariadas para a modelagem dos dados e a predição espacial.

\section{REVISÃO DE LITERATURA}

\section{O setor florestal brasileiro}

Pinus sp., juntamente com outras espécies de rápido crescimento, como, por exemplo, o eucalipto, são importantes matérias-primas para o setor industrial florestal brasileiro, pois, além de gerarem um grande número de empregos, contribuem de maneira expressiva para as exportações do país.

De acordo com o CEPEA-USP/CNA (2006), o PIB Florestal respondeu por 4,1\% do PIB nacional, perfazendo um total US\$ 21 bilhões anuais em 2005, com destaque em três setores: celulose e papel (US\$ 7 bilhões), madeira e móveis (US\$ 9,3 bilhões) e siderurgia a carvão vegetal (US\$ 4,2 bilhões). Com relação à exportação, o setor teve participação com cerca de $10 \%$ do valor total, contribuindo com 5,8 bilhões de dólares no ano de 2005. Devido ao saldo inexpressivo de importações do setor, a economia florestal tem sido, historicamente, responsável por um dos cinco maiores saldos comerciais positivos do país. Quanto à geração de empregos, é responsável por $9 \%$ da população economicamente ativa (sete milhões de pessoas).

O crescimento do segmento do setor de papel e celulose deve ser, em média, de $17 \%$ ao ano no período de 2007 a 2010, em relação aos anos de 2002 a 2005, o que equivale a investimentos para o período da ordem de R $\$ 20$ bilhões, estimulando o desenvolvimento do setor (AGÊNCIA O GLOBO, 2006).

\section{O gênero Pinus}

Devido às condições climáticas, à sua ampla capacidade de adaptação, à sua diversidade de uso e ao seu rápido crescimento, $P$. taeda tem sido uma das espécies preferidas para plantios em reflorestamentos comerciais nos planaltos da região Sul do Brasil, com uma área aproximada de um milhão e oitocentos mil hectares. As seguintes características favorecem o seu plantio: a) a madeira é de cor clara, variando de branca a amarelada; b) é de fibra longa, apropriada para fabricação de papel de alta resistência para embalagens, papel de imprensa e outros tipos de papéis; c) apresenta rusticidade e tolerância, possibilitando o plantio em solos marginais para a agricultura, valorizando assim a terra imprópria para a agricultura convencional, com a produção adicional de madeira, a formação de cobertura 
protetora do solo, a reconstituição de ambiente propício à recomposição espontânea da vegetação nativa em ambientes degradados e a redução por absorção dos índices de carbono da atmosfera (SHIMIZU; MEDRADO, 2006). O povoamento de $P$. taeda é diferenciado pela sua versatilidade, porque, após o corte, sua madeira pode ser destinada à indústria laminadora, que a utiliza para fabricação de compensados, para a indústria de serrados, que a transforma em madeira beneficiada, sendo convertida em móveis, para a indústria de papel e celulose e para a indústria de MDF. Mesmo o seu resíduo tem sido aproveitado como biomassa para geração de vapor e energia (CARGNIN, 2005).

$P$. taeda é conhecido como uma espécie pioneira nas regiões onde ocorre naturalmente (REISSMANN; WISNIEWSKY, 2001). Quanto aos solos, esse gênero de planta é conhecido pela sua baixa exigência nutricional. São encontradas espécies crescendo em sítios extremamente pobres, nos quais a expectativa de produção fica aquém do satisfatório. Os pinheiros, por exemplo, têm revelado uma capacidade extraordinária de gerenciamento dos parcos recursos nutricionais.

As condições de adaptação a solos ácidos, que constituem a grande maioria dos solos dos planaltos do Sul do país, permitiram a implantação de extensas áreas que, juntamente com a adoção de práticas silviculturais adequadas, tornou essa espécie uma importante fonte de matéria-prima, proveniente de florestas estabelecidas dentro dos padrões de sustentabilidade (KRONKA et al., 2005). Essa espécie não cresce bem em locais muito úmidos ou alagados, nem em áreas com solos muito rasos ou erodidos. $\mathrm{Na}$ Planície Costeira Atlântica dos EUA, a produtividade geralmente decresce quanto mais superficial seja o lençol freático (RICHARDSON; RUNDEL, 1998).

Os trabalhos relacionados com a carência nutricional em pinus no Brasil são bastante raros. Segundo Reissmann; Wisniewsky (2001), tal fato se deve principalmente à rapidez de crescimento e à ausência de sintomas de deficiência, especialmente durante as primeiras rotações, reforçando a expectativa de que, de modo geral, a espécie dispense grandes cuidados com adubação.

\section{Análises geoestatísticas}

A modelagem geoespacial permite a descrição quantitativa da variabilidade espacial de atributos de interesse espacialmente distribuídos e a estimativa não tendenciosa da variância mínima de valores desses atributos em locais não amostrados. Avaliar essa variabilidade faz da geoestatística uma eficiente ferramenta de suporte à decisão no manejo de solo e água das espécies florestais. As intervenções para corrigir variações indesejáveis da oferta de nutrientes e água passam a ser balizadas por mapas de isovalores das variáveis de interesse (BOLFE; GOMES, 2007). Mello et al. (2005; 2006) discutem o uso de geoestatística, opções de modelagem e seleção de modelos e métodos para avaliação de inventários florestais em área de eucalipto, adotando, em todos os casos, modelos geoestatísticos univariados.

Ainda segundo Bolfe; Gomes (2007), devem-se analisar e planejar o número de coletas de dados, através do custo/benefício, pois um grande número de pontos amostrais aumenta os custos da operação e pode inviabilizar a implantação do processo. Os métodos geoestatísticos de interpolação, em especial o da krigagem, apresentam propriedades ótimas de estimativas obtidas a partir de dados esparsos. Para a aplicação das técnicas de geoestatística, necessita-se, primeiramente, detalhar a área onde será implantado o estudo. Para tanto, todas as informações devem ser referenciadas juntamente com um processo de amostragem dentro de uma região geográfica definida.

Esse processo é viabilizado pela implantação de um sistema de coordenadas locais ou geográficas, no qual cada atributo ou característica do solo terá suas informações quantitativas e/ou qualitativas (relevo, granulometria, textura, teor de argila, acidez, matéria orgânica etc.) associadas a um ponto no espaço. Essas informações podem ser obtidas por meio de coleta in loco, mapas temáticos, imagens de satélite ou fotografias aéreas. Para referenciar essas informações, utiliza-se o GPS (Sistema de Posicionamento Global), ou, de forma mais simplificada, através da topografia convencional, obtendo-se as coordenadas locais. A teoria das variáveis regionalizadas pressupõe que a variação de uma variável qualquer pode ser expressa pela soma de três componentes: a) uma componente estrutural, associada a um valor médio constante ou a uma tendência; b) uma componente aleatória, espacialmente correlacionada; e c) um ruído aleatório ou erro residual (BURROUGH et al., 1994).

O semivariograma é a principal ferramenta geoestatística para diagnosticar a presença da correlação entre as unidades amostradas (MELLO, 2004). Segundo Ribeiro Jr. (1995), os semivariogramas são preferidos para caracterizar a estrutura de continuidade espacial da característica avaliada por exigirem hipóteses de estacionaridade menos restritivas (hipótese intrínseca). É esperado que observações mais próximas geograficamente tenham comportamentos mais semelhantes entre si do que 
aquelas separadas por maiores distâncias. Dessa maneira, é esperado que a semivariância $\gamma(\mu)$ aumente com a distância $\mu$.

O parâmetro alcance $(\varnothing)$ controla o crescimento de $\gamma(\mu)$, sendo importante para a interpretação dos semivariogramas. Ele está relacionado com a distância até onde os pontos amostrais estão correlacionados entre si (VIEIRA et al., 1983; VIEIRA, 1997), ou seja, os pontos localizados em uma área cujo raio seja o alcance são mais semelhantes entre si do que os separados por distâncias maiores. Segundo McBratney; Webster (1986) e Vieira et al. (2002), uma das principais utilizações dos semivariogramas é na determinação do número ideal de amostras para a estimação de características químicas, físicas ou físico-hídricas do solo, separadas por distâncias que as tornam independentes entre si.

Conhecido o semivariograma e havendo dependência espacial entre as amostras, podem-se interpolar valores em qualquer posição no campo de estudo, sem tendência e com variação mínima. Inúmeros métodos de interpolação univariados e multivariados, com diversos níveis de complexidade, estão disponíveis na literatura (GOOVAERTS, 1997; CARVALHO; ASSAD, 2002). Ainda, o estudo da variabilidade espacial das características do meio físico é uma etapa importante para a silvicultura de precisão. Nessa fase, a utilização do semivariograma permite planejar desenhos ótimos de amostragem, com o intuito de capturar a variabilidade em escalas espaciais em até poucos metros, e a estimação espacial usando krigagem permite obter mapas da distribuição espacial de cada característica do solo, por exemplo.

A técnica geoestatística da krigagem é considerada uma metodologia de interpolação de dados. Ela utiliza o dado tabular e sua posição geográfica para calcular as interpolações. Utilizando o princípio da Primeira Lei de Geografia de Tobler, que diz que unidades de análise mais próximas entre si são mais parecidas do que unidades mais afastadas, a krigagem utiliza funções matemáticas para acrescentar pesos maiores nas posições mais próximas aos pontos amostrais e pesos menores nas posições mais distantes, e criar assim os novos pontos interpolados com base nessas combinações lineares de dados (JAKOB, 2002). Além disso, o que diferencia a krigagem de outros métodos de interpolação é a estimação de uma matriz de covariância espacial que determina os pesos atribuídos às diferentes amostras, o tratamento da redundância dos dados, a vizinhança a ser considerada no procedimento inferencial e o erro associado ao valor estimado.

A krigagem também fornece estimadores exatos com propriedades de não-tendenciosidade e eficiência (CARVALHO; ASSAD, 2002). Os métodos de krigagem usam a dependência espacial entre amostras vizinhas, expressas no semivariograma, para estimar valores em qualquer posição dentro do campo, sem tendência e com variância mínima, ou seja, são estimadores ótimos muito usados no estudo da distribuição espacial de atributos do solo (VIEIRA, 2000), da precipitação pluvial etc. (CARVALHO; ASSAD, 2002).

A estimação dos parâmetros do modelo para subseqüente uso na predição espacial pode ser baseada em ajustes de funções teóricas ao variograma empírico, ou ajustando-se um modelo diretamente aos dados, adotando-se estimadores verossimilhança métodos bayesianos de inferência. Cressie (1993) apresenta diversos métodos para estimação dos parâmetros. Diggle; Ribeiro Jr. (2002) detalham e apresentam uma implementação computacional de métodos baeysianos de inferência para modelos gaussianos, enquanto que Diggle; Ribeiro Jr. (2002) adotam e descrevem métodos baseados na função de verossimilhança, incluindo bayesianos no contexto de dados gaussianos, gaussianos transformados e nãogaussianos com distribuições na família exponencial.

No caso de observações bi ou multivariadas, os modelos e procedimentos são estendidos de diferentes formas, visando modelar as covariâncias simples e cruzadas espaciais entre as variáveis envolvidas (WACKERNAGEL, 2003). Uma possível estratégia para isso é a adoção do modelo de corregionalização linear descrito, por exemplo, em Goovaerts (1997). A partir do ajuste de tal modelo é possível obter predições espaciais das variáveis de interesse através do procedimento conhecido como cokrigagem, no qual o valor predito em um determinado ponto é dado por uma média ponderada dos dados das variáveis envolvidas.

Segundo Isaaks; Srivastava (1989), a cokrigagem leva em consideração uma ou mais variáveis secundárias na estimativa de uma variável primária, e poderá ser escrita em termos das covariâncias simples e cruzadas, sendo os pesos $W$ usados na interpolação dados por $W=C^{-1} D$, em que:

$$
C=\left(\begin{array}{cccc}
\operatorname{Cov}\left(\gamma_{1} \gamma_{1}\right) & \operatorname{Cov}\left(\gamma_{1} \gamma_{2}\right) & 1 \\
\operatorname{Cov}\left(\gamma_{2} \gamma_{1}\right) & \operatorname{Cov} & \left(\gamma_{2} \gamma_{2}\right) & 1 \\
1 & 0 & 0 \\
0 & 1 & 0
\end{array}\right) \quad W=\left(\begin{array}{c}
a \\
\tilde{b} \\
\tilde{\sim} \lambda_{1} \\
-\lambda_{2}
\end{array}\right) \quad D=\left(\begin{array}{cc}
\operatorname{Cov}\left(\gamma_{1} \gamma_{0}\right) \\
\operatorname{Cov}\left(\gamma_{2} \gamma_{0}\right) \\
1 \\
0
\end{array}\right)
$$


Onde: $\gamma_{1}$ é a variável primária, $\gamma_{2}$ a variável secundária, $\gamma_{0}$ os valores a serem preditos, ${ }_{\sim}^{a} \mathrm{e} \underset{\sim}{b}$ são os vetores de pesos atribuídos às variáveis primária e secundária, respectivamente, tais que $\sum_{i=1}^{m_{1}} a_{i}=1 \mathrm{e} \sum_{i=1}^{m_{1}} b_{i}=0$ são restrições para garantir a não-tendenciosidade, introduzidas pelo uso dos operadores de Lagrange $\lambda_{1}$ e $\lambda_{2}$.

\section{MATERIAL E MÉTODOS}

\section{Localização do experimento}

O experimento situa-se numa área efetiva de plantio de 2.252 ha em área de reflorestamento de P. taeda, da Empresa Modo Batistella de Reflorestamento S/A (MOBASA), situada no estado de Santa Catarina, na divisa entre os municípios de Rio Negrinho e Doutor Pedrinho (Figura 1). O microclima da região pertence à classe $\mathrm{Cfa}$ da classificação de Köppen, que é tipicamente subtropical de verão quente, sem estação seca de inverno, com temperaturas médias do mês mais frio entre $-3{ }^{\circ} \mathrm{C}$ e $18{ }^{\circ} \mathrm{C}$. A região caracteriza-se ainda por possuir elevada pluviosidade, bem distribuída durante o ano, e por não apresentar estação seca definida.

A classe de solo predominante é a dos Cambissolos Húmicos Alumínicos típicos ou lépticos (EMBRAPA, 2006). Esses solos são medianamente profundos, com teores de matéria orgânica superiores a 40 g. $\mathrm{dm}^{-3}$ no horizonte superficial (normalmente em torno de $40-50 \mathrm{~cm}$ de espessura), além de extremamente ácidos e com teores de alumínio trocável superiores a $4,0 \mathrm{cmol}_{\mathrm{c}} \cdot \mathrm{dm}^{-3}$.

\section{Amostragem}

A base de dados utilizada neste estudo é proveniente de povoamentos comerciais de $P$. taeda, coletados em parcelas de inventário florestal contínuo, com área de $500 \mathrm{~m}^{2}$ e espaçamento entre árvores de 2,80 x 2,80 m. Em cada parcela, foi determinada a caracterização física do seu solo, com medidas dos teores de argila das camadas superficial $(0-20 \mathrm{~cm})$ e subsuperficial $(30-50 \mathrm{~cm})$ (Figura 2).

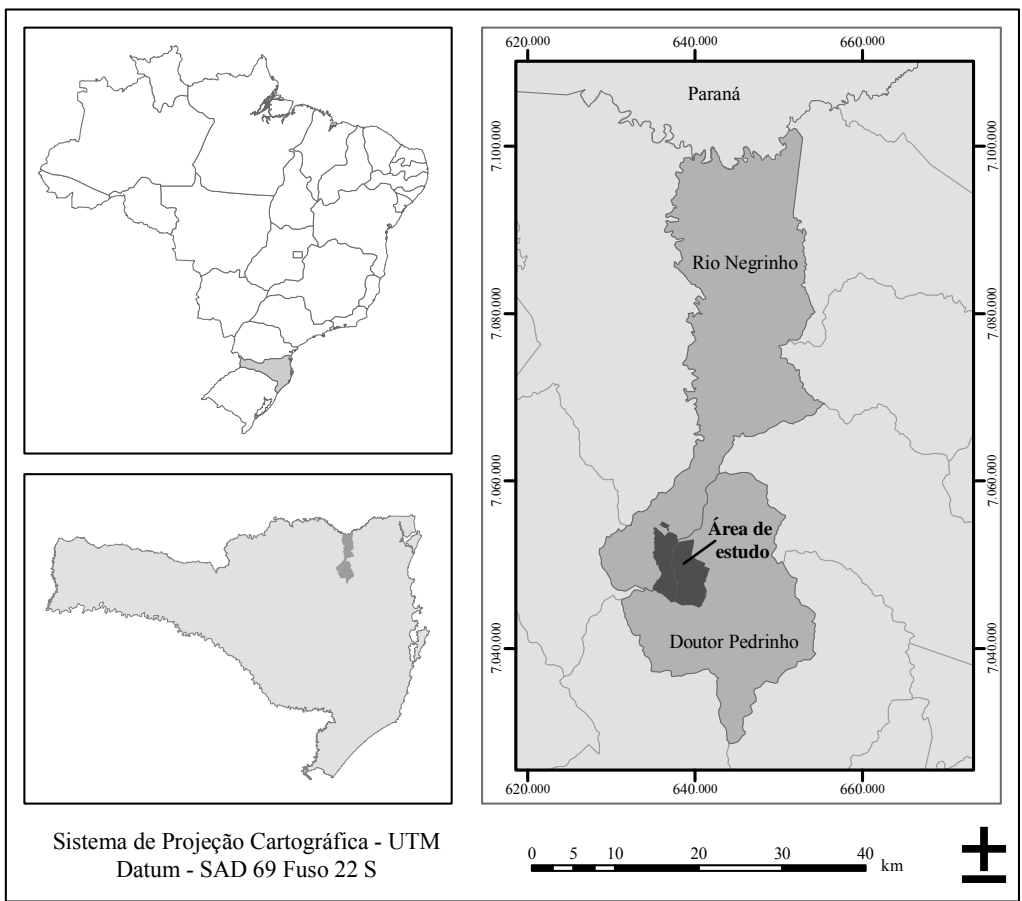

Fonte: MOBASA - setor SIG

Figura 1. Localização da área de estudo.

Figure 1. Location of the study area. 
Foram obtidas medidas de crescimento das árvores em cinco idades diferentes: 11 a 15 anos, sumarizadas através do incremento anual volumétrico (IMA). Como o objetivo deste trabalho foi realizar predições, em locais não amostrados, de uma variável com tamanho pequeno de amostra, foram medidos em 18 coordenadas (primeiro grupo amostral), aleatoriamente distribuídas na área, além de outros atributos, os teores de argila e o IMA. Além disso, visando ampliar a qualidade da informação espacial na área, foram tomadas em mais 555 coordenadas (segundo grupo amostral), também casualmente distribuídas, medidas de concentração de argila, por apresentar certo grau de associabilidade com o IMA (Figura 3).

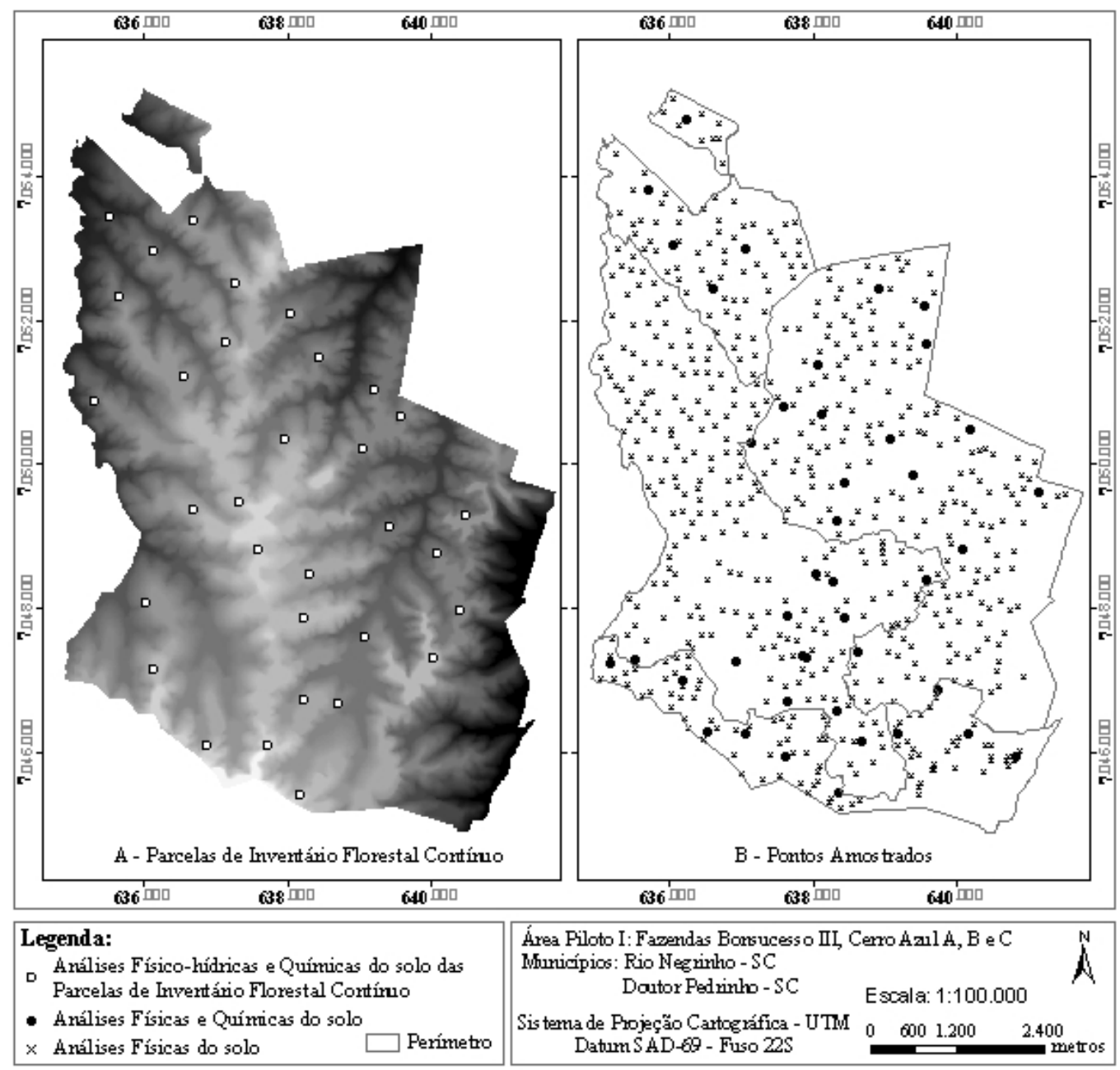

Figura 2. Localização das amostras de solos e das parcelas de inventários florestais contínuos na área de estudo.

Figure 2. Location of the soil samples and of the sample plots of continuous forest inventory within the study area.

\section{Metodologia de análise geoestatística}

A idéia central foi obter mapas que descrevessem o comportamento de uma variável de interesse na área de estudo, através de predições em locais não amostrados. Nesse caso, a variável primária possui tamanho de amostra pequeno, mas está correlacionada com outra variável com amostra de tamanho maior. Foi considerada a variável principal, ou primária, o IMA, e para ela pretendeu-se construir o mapa de distribuição espacial. Como variável suporte ou secundária, adotou-se a argila. Para se avaliar a correspondência entre elas, foi construído, para os pares de valores medidos no conjunto das 18 coordenadas amostrais, um diagrama de dispersão e efetuado um teste de Correlação de Pearson. 


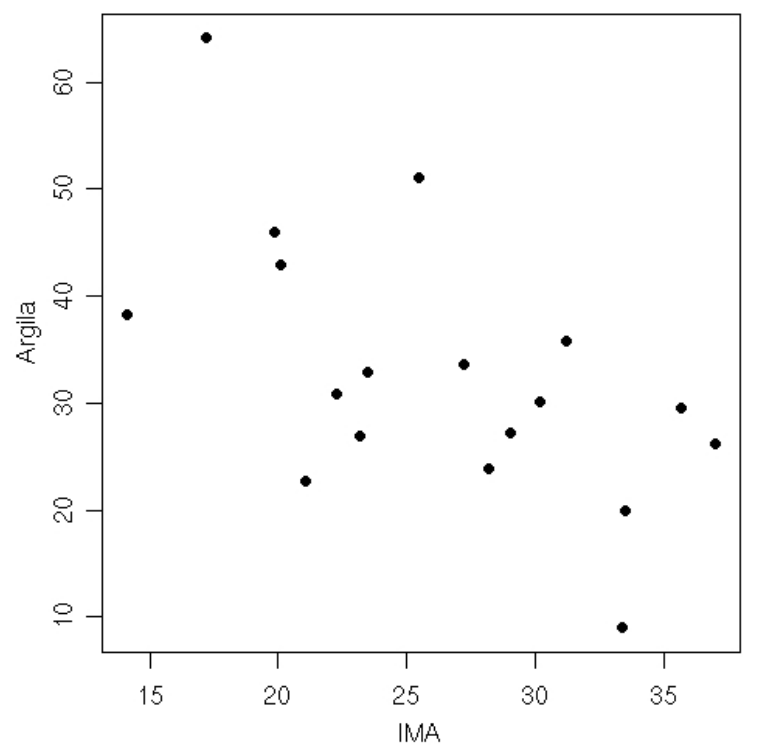

Figura 3. Diagrama de dispersão da argila versus IMA.

Figure 3. Dispersion diagram of the clay versus IMA.

Segundo Diggle; Ribeiro Jr. (2007), o formato básico dos dados geoestatísticos multivariados é dado por $(x, y)$, onde $x$ é o conjunto de $n$ coordenadas amostrais na área de um espaço bidimensional e $y$ indica uma medida escalar (caso univariado) ou vetorial (caso multivariado) tomada em cada coordenada $x$. Esse conjunto de medidas representa um número finito de observações de uma realização de um processo estocástico gaussiano $S(x)$ desconhecido. Dessa forma, a variável aleatória observável $Y$, que irá depender da localização $x$, foi modelada como: $Y\left(x_{i}\right)=\mu\left(x_{i}\right)+S\left(x_{i}\right)+\delta_{i}, \quad i: 1,2, \ldots, n . Y\left(x_{i}\right) \sim N\left(\mu\left(x_{i}\right), \tau^{2}\right)$, e $\mu\left(x_{i}\right)$ é uma tendência espacial externa ao processo. Para modelos com média constante $\mu\left(x_{i}\right)=\mu$, o processo é definido como estacionário na média. $S\left(x_{i}\right)$ é um processo gaussiano multivariado com média zero e variância $\sigma^{2} \mathrm{e}$ função de correlação que caracteriza o processo, e os $\delta_{i} \sim N\left(0 ; \tau^{2}\right)$ são os erros aleatórios independentes.

Neste trabalho, consideraram-se os casos uni e bivariados estacionários, em que a variável $Y_{1}$ representou o processo gaussiano do IMA tomado nas 18 coordenadas do primeiro grupo amostral e $Y_{2}$ representou o processo gaussiano da concentração de argila nas 555 coordenadas do segundo grupo amostral. Assim, a análise geoestatística da variável IMA é fisicamente restrita, por constituir um dado de difícil observação e muito dispendioso de ser obtido. Como inferências com pequenas amostras são pouco confiáveis, contornou-se esse problema obtendo-se, em outras localizações, medidas de concentração de argila (variável secundária), que é de mais fácil obtenção, permitindo expandir informações da variável principal. A natureza desse processo bivariado foi modelado como:

$$
\begin{array}{ll}
\gamma_{1, i}=\mu_{1}+\sigma_{01} R_{0}\left(x_{i}\right)+\sigma_{1} R_{1}\left(x_{i}\right) & i=1,2, \ldots, n \\
\gamma_{2, j}=\mu_{2}+\sigma_{02} R_{0}\left(x_{j}\right)+\sigma_{2} R_{2}\left(x_{j}\right) & j=1,2, \ldots, n
\end{array}
$$

Nessa formulação, $\sigma R\left(x_{i}\right)=S\left(x_{i}\right)$ (DIGGLE; RIBEIRO JR., 2007), sendo $R(x)$ a matriz de correlação. $R_{0}(x)$ é um processo subjacente às duas variáveis, $R_{I}(x)$ é específico ao processo $Y_{I}$ e $R_{2}(x)$ é específico ao processo $Y_{2}$. A função de correlação presente na matriz de correlação que adotamos foi a dada pelo modelo de Mátern, devido à flexibilidade que ela oferece para modelar o decaimento da correlação, bem como a suavidade do processo, através da modificação de seus parâmetros. A função de 
correlação de Matérn é dada por: $\rho(\mu, \phi, k)=\left(2^{k-1}\right)^{-1}\left(\frac{\mu}{\phi}\right) K_{k}\left(\frac{\mu}{\phi}\right)$, onde o alcance ( $\phi$ ) é um parâmetro de decaimento da função em relação à distância $\mu \mathrm{e}_{k}$ é um parâmetro de diferenciabilidade da função na origem e, portanto, estabelece a suavidade do processo. $K$ é a função modificada de Bessel do terceiro tipo (ABRAMOWITZ; STEGUN, 1965).

Para o modelo bivariado proposto, foi então necessário estimar os $\mu_{1} \mu_{2} \sigma_{01} \sigma_{02} \sigma_{1} \sigma_{2} \phi_{0}$ (em $R_{0}$ ), $\phi_{1} \phi_{2}$ (em $R_{1} R_{2}$ ) e $\tau_{1} \tau_{2}$. O método empregado foi o da maximização da função logverossimilhança, dada por:

$$
\ell\left(\mu, \tau^{2}, \sigma^{2}, \phi\right) \alpha-\frac{n}{2} \log \left(\sigma^{2} R(\phi)+\tau^{2} I\right)-\frac{1}{2}(y-1)\left(\sigma_{\sim}^{2} R(\phi)+\left(\tau^{2} I\right)^{-1}(y-\mu)\right.
$$

No caso univariado, a matriz de correlação $R(\phi)$ foi constituída pelos elementos $\left[\rho\left(u_{i j}\right)\right.$ ], que correspondem às correlações entre as coordenadas $x_{i}$ e $x_{j}$ para $i \neq j$, e corresponde ao valor unitário para $i$ $=j$. Já no caso bivariado, essa matriz foi dada por:

$$
\left(\begin{array}{ll}
\operatorname{Cor}\left(Y_{1}, Y_{1}\right) & \operatorname{Cor}\left(Y_{1}, Y_{2}\right) \\
\operatorname{Corr}\left(Y_{2}, Y_{1}\right) & \operatorname{Corr}\left(Y_{2}, Y_{2}\right)
\end{array}\right)
$$

Nessa matriz, os elementos da diagonal principal representam as matrizes de autocorrelações das variáveis $\mathrm{Y}_{1}$ e $\mathrm{Y}_{2}$. Já os elementos da diagonal secundária correspondem a matrizes de correlações cruzadas.

Para a estimação univariada de valores de IMA em locais não amostrados, compatíveis com a produção de um mapa temático, foi construído um grid de predição contendo 7.176 coordenadas. Nele foi construído um mapa associando os valores estimados em cada coordenada $x_{0}$ a uma tonalidade de cinza dentro de uma escala apropriada.

O método de estimação foi aquele sugerido por Stein (1999), Schabenberger; Gotway (2005) e Diggle; Ribeiro Jr. (2007), dado por: $E\left(\widehat{Y}_{1}\left(x_{0}\right) / Y_{1}\right)=\widehat{\mu}+r^{\prime}\left(\widehat{\sigma}^{2} R(\widehat{\phi})+\widehat{\tau}^{2} I\right)^{-1}(y-\widehat{\mu} I)$, onde $r$ representa o vetor de correlação entre a coordenada alvo $x_{0}$ e cada uma das 18 coordenadas tomadas para a variável IMA. Já a estimação bivariada para IMA em 18 coordenadas, contando com informações espaciais da variável argila, tomadas em 555 coordenadas, foi feita por:

$$
E\left(\hat{Y}_{1}\left(x_{0}\right) / Y_{1} ; Y_{2}\right)=\hat{\mu}+r^{\prime}\left(\hat{\sigma}^{2} R(\hat{\phi})+\bar{\tau}^{2} I\right)^{-1}(y-\widehat{\mu} I) .
$$

Nesse caso, a matriz de correlações $R(\phi)$ foi constituída pelas matrizes de autocorrelações e de correlações cruzadas, y contém as 18 observações da variável IMA seguida das 555 observações da variável argila, $\hat{\mu}, \widehat{\sigma}, \widehat{\phi}, \widehat{\tau}$ assumem os valores estimados para o modelo, relativamente à sua variável correspondente.

Para os cálculos estatísticos e geoestatísticos e para a produção dos gráficos, empregou-se o programa estatístico R e o pacote geoR (RIBEIRO JR.; DIGGLE, 2001), ambos os programas livres dentro da licença internacional GPL (General Public Licence).

\section{RESULTADOS E DISCUSSÃO}

Na primeira fase do processo de análise, buscou-se estabelecer medidas quantitativas para cada uma das variáveis. A tabela 1 mostra que a variável IMA, tomada em 18 coordenadas, apresentou uma média de $26,36 \mathrm{~m}^{3}$ e um desvio padrão de $6,55 \mathrm{~m}^{3}$, resultando em um coeficiente de variação $(\mathrm{CV}) \mathrm{de}$ $24,8 \%$, tido como baixo (valores relativamente homogêneos).

O teste de Shapiro; Wilk (1965) resultou em p-valor de 0,917, revelando distribuição normal homocedástica para essa variável, não havendo a necessidade de sua transformação. Já a variável argila, nessas mesmas coordenadas, apresentou média de $32,84 \%$ e desvio padrão $12,54 \%$, resultando um coeficiente de variação (CV) de $38,2 \%$, indicando uma dispersão dos seus valores em torno da média um pouco mais acentuada. $\mathrm{O}$ teste de normalidade para essa variável resultou em p-valor de 0,5 , não sugerindo necessidade de transformação. Essa mesma variável (argila), tomada em 555 coordenadas, resultou na média de $25,19 \%$ com desvio padrão de $7,78 \%$ e coeficiente de variação de $30,9 \%$, 
compatível, portanto, com o resultado anterior. O teste de normalidade resultou em p-valor próximo de zero, sugerindo necessidade de transformação.

Tabela 1. Medidas quantitativas das variáveis em estudo.

Table 1. Quantitative measures of the variables in study.

\begin{tabular}{lcccc}
\hline Variável & $\mathbf{N}^{\circ}$ Obs. & Média & D.P. $(\%)$ & S.W. (p-valor) \\
\hline IMA $\left(\mathrm{m}^{3}\right)$ & 18 & 26,36 & 24,8 & 0,917 \\
Argila (\%) & 18 & 32,84 & 38,2 & 0,502 \\
Argila (\%) & 555 & 25,19 & 25,19 & 0,000 \\
\hline
\end{tabular}

D.P.: desvio padrão; S.W.: teste de Shapiro; Wilk (1965).

O teste de Correlação de Pearson para medir a associabilidade entre a variável primária (IMA) e a variável secundária (argila) resultou numa relação linear inversa entre elas de $-0,59$, com $0,1 \%$ de significância, ou seja, um aumento nos teores de argila leva a uma redução no IMA. O diagrama de dispersão da figura 3 , permite uma melhor visualização da distribuição dos dados dessa correlação.

Para a análise geoestatística univariada, procurou-se ajustar um modelo de correlação espacial para cada variável. Segundo a tabela 2, o modelo exponencial ajustado à estrutura de correlação espacial da variável IMA tomada em 18 coordenadas não exigiu transformações dos dados dessa variável, e dela em função da argila $(\lambda=1)$. Diggle; Ribeiro Jr. (2002) discutem o uso da família Box-Cox de transformações (BOX e COX, 1964) em modelos geoestatísticos. O coeficiente de efeito pepita (CEP), dado pela razão entre $\tau^{2}$ e $\tau^{2}+\sigma^{2}$, sugere uma estrutura de dependência espacial até uma distância de $476,42 \mathrm{~m}$. Na prática, o alcance é três vezes o seu valor definido, ou seja, é de $1.428 \mathrm{~m}$. O valor de $\tau^{2}=0$ indica a ausência de variação de pequena escala.

Quando foi incorporada no modelo a argila como co-variável, o melhor modelo obtido foi, ainda, o exponencial, sem necessidade de transformação dos dados. Entretanto, devido à estimativa $\sigma^{2}$ ter resultado nula, o CEP indicou um efeito pepita puro, ou seja, ausência de estrutura de dependência espacial. Tal afirmação é corroborada pela estimativa nula do parâmetro de alcance $\varnothing$. O resultado é natural, devido à forma de correlação entre as variáveis.

A estrutura de correlação espacial para a variável argila com 555 observações indicou, após uma transformação logarítmica nos dados, exigido pelo parâmetro $\lambda=0$, um modelo exponencial.

$O$ coeficiente de efeito pepita sugere uma estrutura variográfica até o alcance de $\varnothing=2.007$, o que equivale a $6.021 \mathrm{~m}$ de dependência espacial. $\tau^{2}$ também indicou uma pequena variância de pequena escala.

Tabela 2. Modelo exponencial ajustado à estrutura de correlação espacial da variável IMA.

Table 2. Exponential model fitted to the structure of spatial correlation of the variable IMA.

\begin{tabular}{lcccccccccc}
\hline Variável & $\mathbf{N}^{\mathbf{0}}$ Obs. & $\beta$ & $\tau^{2}$ & $\sigma^{2}$ & $\varnothing$ & $\lambda$ & MOD & K & -MLV & CEP \\
\hline IMA & 18 & 26,69 & 0 & 39,57 & 476,42 & 1 & Expon. & 0,5 & 58,4 & 0 \\
IMA+Argila & 18 & $36,34 /-0,31$ & 26,37 & 0 & 0 & 1 & Expon. & 0,5 & 54,99 & 1 \\
Argila & 555 & 3,08 & 0,03 & 0,18 & 2006,99 & 0 & Expon. & 0,5 & 1765 & 0,12 \\
\hline
\end{tabular}

Para uma análise bivariada, utilizando a argila tomada em 555 coordenadas, para ampliar as estimativas de IMA, obtiveram-se, conforme o modelo (4), as estimativas de máxima verossimilhança: $\mu_{1}$ $=25,50, \mu_{2}=23,98, \quad \sigma_{01}^{2}=9,31, \sigma_{02}^{2}=50,48, \sigma_{1}^{2}=103,29, \sigma_{2}^{2}=0,05, \varnothing_{0}=722,62, \varnothing_{1}=$ $1.488,71, \varnothing_{2}=1.675,18$.

Com os resultados desses modelos, foi possível, por krigagem ordinária, produzir o mapa de predição de IMA para a área (Figura 4A), utilizando-se 18 pontos amostrais originais, dando um IMA médio de 26,69 $\mathrm{m}^{3} \cdot \mathrm{ha}^{-1} \cdot$ ano $^{-1}$ e um desvio padrão de krigagem de $6,20 \mathrm{~m}^{3} \cdot \mathrm{ha}^{-1} \cdot$ ano $^{-1}$. Já o mapa do IMA (Figura 4B) foi produzido utilizando-se as co-estimativas médias adequadas.

A preocupação em amostrar adequadamente florestas, conciliando precisão de estimativas com custos realísticos na prática, é tema de interesse constante na literatura de avaliação de inventários, com os pesquisadores tendo se preocupado com métodos de amostragem eficientes e de baixo custo. $\mathrm{O}$ 
inventário florestal tem por objetivo aplicar e avaliar métodos de amostragem que gerem resultados mais precisos. O desejável para todo empreendimento florestal é, portanto, obter informações precisas e a baixo custo sobre a produção florestal. A redução do custo no inventário florestal está alicerçada basicamente na redução da intensidade amostral (MELLO, 2004).
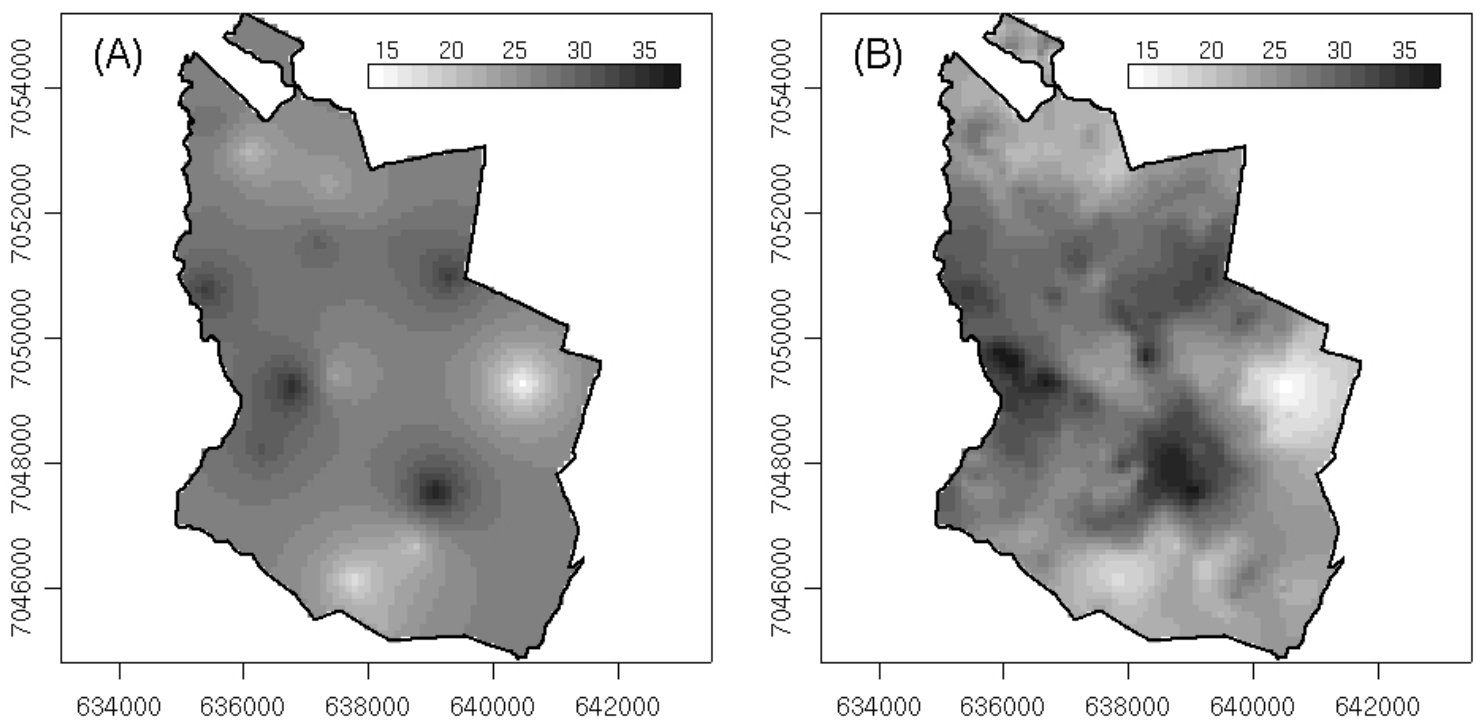

Figura 4. Mapa de distribuição espacial do IMA com valores preditos por krigagem (A) a partir da amostra de 18 pontos de IMA e preditos com cokrigagem (B) utilizando também os 555 dados de teores de argila.

Figure 4. Map of spatial distribution of IMA with values predicted by kriging (A) from 18 IMA sample points and (B) predicted by cokriging adding the 555 clay samples.

Dessa forma, devido à presença de continuidade espacial no presente estudo, o uso do estimador geoestatístico multivariado, para estimativa do incremento médio anual volumétrico (IMA) de $P$. taeda, foi preferido ao estimador geoestatístico usual univariado, por duas razões: 1) proporcionou estimativas mais detalhadas da variável de interesse, mantendo a propriedade de ausência de viés; e 2) é de mais baixo custo quando comparado ao estimador clássico (krigagem ordinária), em vista de se obterem os teores de argila através do mapeamento detalhado dos solos de forma mais econômica do que os levantamentos dendrométricos de pré-corte, praticados pelas empresas florestais madeireiras.

\section{CONCLUSÕES}

Através do modelo geoestatístico avaliado e ajustado pelo método da máxima verossimilhança, foi possível produzir mapas de predição de "IMA volumétrico" para a espécie Pinus taeda L., influenciado pelos teores de argila, quer por krigagem, quer por co-krigagem ordinária, resultando em estimativas médias adequadas.

O desenvolvimento deste trabalho permitiu verificar que a característica dendrométrica avaliada no inventário florestal (IMA) apresentou-se estruturada espacialmente. Esse resultado sugere que, no processamento do inventário florestal, na presença de estrutura de continuidade espacial, as parcelas (unidades amostrais) não devem ser tratadas de forma independente.

As empresas florestais, de maneira geral, conduzem um grupo de parcelas permanentes nos projetos florestais implantados, permitindo a avaliação do crescimento da floresta. Na idade de corte da floresta, é preciso estimar o volume de cada talhão que compõe os projetos, uma vez que somente as informações das parcelas permanentes não proporcionam estimativas precisas por talhão. É necessário, portanto, efetuar o inventário pré-corte, o qual lança uma intensidade amostral maior em cada talhão. Assim, há um novo custo adicionado no processo de medição da floresta. Fazendo uso do estimador 
geoestatístico multivariado, o qual considera a obtenção de uma variável de menor custo, a estimativa de uma outra variável dependente e altamente correlacionáveis, é possível melhorar a precisão a estimativa volumétrica por talhão fazendo uso somente das parcelas permanentes. Além disso, explorar metodologias multivariadas pode reduzir custos amostrais de variáveis cujas medidas sejam dispendiosas, mantendo-se a precisão das estimativas ao se explorarem as relações espaciais entre diferentes variáveis.

Em síntese, a aplicação da teoria clássica de amostragem no inventário florestal pode aumentar os problemas estatísticos, caso haja estrutura de continuidade espacial. Assim, é necessário, primeiramente, na análise do inventário florestal, verificar se há ou não estrutura de continuidade espacial da característica avaliada. Em se confirmando, deve-se fazer uso dos benefícios dela através da introdução da componente espacial no modelo. Conclui-se, ao se analisar o conjunto dos resultados obtidos pelas técnicas geoestatísticas utilizadas, que esse conjunto foi coerente com os conhecimentos da ciência florestal e que, portanto, têm grande potencial para uso nesse segmento, e a metodologia testada aqui pode servir de ponto de partida para novas propostas que explorem estruturas multivariadas espaciais adequadas a problemas florestais.

\section{AGRADECIMENTOS}

À Empresa Madeireira Modo Battistella Reflorestamento S.A., Rio Negrinho (SC), pelo apoio logístico e financeiro para o desenvolvimento da pesquisa.

\section{REFERÊNCIAS}

ABRAMOWITZ, M.; STEGUN, I. Handbook of mathematical functions. $9^{\text {th }}$ ed. New York:Dover, 1965.

AGÊNCIA O GLOBO. Impactos sobre a biodiversidade. Disponível em: <http://www.mma.gov.br>. Acesso em: 18/10/2006.

BOLFE, E. L.; GOMES, J. B. V. Geoestatística como subsídio à implantação de agricultura de precisão. Disponível em: <http://www.agronline.com.br/artigos/artigo.php?id=210>. Acesso em: 23/01/2007.

BOX, G. E. P; COX, D. R. An analysis of transformations (with Discussion). Journal of the Royal Statistical Society. Methodological. Series B, London, v. 26, p. 211-252, 1964.

BURROUGH, P. A.; BOUMA, J.; YATES, S. R. The state of the art in pedometrics. Geoderma, Amsterdam, v. 62, n. 1/3, p. 311-326, 1994.

CARGNIN, O. Alternativas das florestas de pinus. [on line]. 2005. Disponível em: $\leq$ http://www.valeverde.org.br/html/clipp2.php?id=3752\&categoria=Biodiversidade $>$. Acesso em: 15/08/06.

CARVALHO, J. R. P. de; ASSAD, E. D. Comparação de interpoladores espaciais univariados para precipitação pluvial anual no estado de São Paulo. Campinas: Embrapa Informática Agropecuária, 2002. 6 p. (Comunicado Técnico, n. 33). Mapa.

CEPEA/USP - Centro de Estudos Avançados em Economia Aplicada - Escola Superior de Agricultura Luiz de Queiroz. Estatísticas. Disponível em: <http://www. cepea.esalq.usp.br/florestal/>. Acesso em: 18/10/2006.

CRESSIE, N. Statistics for spatial data. Rev. ed. New York: J. Wiley \& Sons, 1993.

DIGGLE, P. J.; RIBEIRO JR, P. J. Bayesian Inference in Gaussian model based geostatistics. Geographical And Environmental Modelling, Oxfordshire, v. 6, n. 2, p. 129-146, 2002.

DIGGLE, P.J.; RIBEIRO JÚNIOR, P.J. Model-based geostatistics. New York: Springer, 2007. 228 p. 
EMBRAPA - Empresa Brasileira de Pesquisa Agropecuária. Centro Nacional de Pesquisa de solos. Sistema brasileiro de classificação de solos. $2^{a}$ ed. rev. Brasília, DF: EMBRAPA-SPI; Rio de Janeiro: EMBRAPA-CNPS, 2006. 306 p.

GOOVAERTS, P. Geostatistics in soil science: state of the art and perspectives. Geoderma: an international journal of soil science, Amsterdam, v. 89, p.1-45, 1997.

ISAAKS, E. H.; SRIVASTAVA, R. M. An Introduction to applied geostatistics. New York: Oxford University Press, 1989. 560 p.

JAKOB, A. A. E. Kriging demographic variables in order to look for trends in the spatial distribution of population. Trabalho final do curso Spatial Modeling and Analysis - CSS 620. Ithaca: Department of Crop and Soil Sciences, Cornell University, 2002.

KRIGE, D. G. Two-dimensional weighted moving average trend surfaces for ore-evaluation. South African Institute of Mining and Metallurgy. Journal, Johannesburg, v. 66, p. 13-38, 1966.

KRONKA, F. J. N.; BeRTOlAni, F.; PONCE, R. H. A cultura do Pinus no Brasil. Disponível em: $<$ http://www.sbs.org.br/atualidades_single.php?id=4467>. Acesso em: 18/10/2006.

MATHERON, G. The theory of regionalized variables and its applications. Fontainebleau: École Nationale Supérieux das Mines de Paris. 1971. 211 p. (Les Cahiers de Centre de Morphologie Mathématique de Fontainebleau, n. 5).

MCBRATNEY, A. G.; WEBSTER, A. G. Choosing functions for semi-variograms and fitting them to sampling estimates. The Journal of Soil Science, Oxford, GB, v.,37, p.,617-639, 1986.

MELLO, J. M. de. Geoestatística aplicada ao inventário florestal. 122 p. Dissertação (Doutorado) Escola Superior de Agricultura "Luiz de Queiroz”, Universidade de São Paulo, Piracicaba, 2004.

MELLO, J. M.; BATISTA, J. L. F.; RIBEIRO JÚNIOR, P. J.; OLIVEIRA, M. S. Ajuste e seleção de modelos espaciais de semivariograma visando à estimativa volumétrica de Eucalyptus grandis. Scientia Forestalis, Piracicaba, v. 69, p. 25-37, 2005.

MELLO, J. M.; OLIVEIRA, M. S.; BATISTA, J. L. F.; RIBEIRO JÚNIOR, P. J.; KANEGAE JUNIOR, H. Uso do estimador geoestatístico para predição volumétrica por talhão. Floresta, Curitiba, v. 36, p. 251-260, 2006.

REISSMANN, C. B.; WISNIEWSKI, C. Aspectos nutricionais de plantios de Pinus. Curitiba: UFPR, 2001. Disponível em: <http://agrarias.ufpr.br/ mrlima/pesquisas/ R001.htm>. Acesso em: 23/01/2006.

RIBEIRO JÚNIOR, P. J. Métodos geoestatísticos no estudo da variabilidade espacial de parâmetros do solo. 99 p. Dissertação (Mestrado) - Escola Superior de Agricultura "Luiz de Queiroz", Universidade de São Paulo, Piracicaba, 1995.

RIBEIRO JÚNIOR, P. J.; DIGGLE, P. J. GeoR: a package for geostatistical analysis. R-NEWS, v. 1, n. 2, p. 15-18, 2001.

RICHARDSON, D. M.; RUNDEL, P. W. Ecology and biogeography of pinus: an introduction. In: RICHARDSON, D. M. (Ed.). Ecology and biogeography of Pinus. New York: Cambridge University Press. Cambridge, p.1-48, 1998.

SCHABENBERGER, O.; GOTWAY, A. Statistical methods for spatial data analysis, New York: ChapmanHall, 2005.

SHAPIRO, S. S.; WILK, M. B. An analysis of variance test for normality (complete samples). Biometrika, London, v. 53, n. 3/4, p. 591-611, 1965.

SHIMIZU, J. Y.; MEDRADO, M. J. S. Cultivo do Pinus. Disponível em: $<$ http://sistemasdeproducao.cnptia.embrapa.br/FontesHTML/Pinus/CultivodoPinus/index.htm>. Acesso em: 18/10/2006. 
STEIN, M. L. Interpolation of spatial data: some theory for kriging. New York: Springer series in statistics. 1999.

TRANGMAR, B. B.; YOST, R. S.; UEHARA, G. Application of geostatistics to spatial studies of soil properties. Advances in Agronomy, San Diego, Calif., v. 38, p. 45-94, 1985.

VIEIRA, S. R. Variabilidade espacial de argila, silte e atributos químicos em uma parcela experimental de um latossolo roxo de Campinas (SP). Bragantia, Campinas, v. 56, n. 1, p. 1-17, 1997.

VIEIRA, S. R. Geoestatística em estudos de variabilidade espacial do solo. In: NOVAES, R. S.; ALVAREZ V, V. H.; SCHAESER, C. E. G. R. (Eds.). Tópicos em ciências do solo. Viçosa, MG: Sociedade Brasileira de Ciência do Solo, 2000. p. 1-54.

VIEIRA, S. R.; HATFIELD, J. L.; NIELSEN, D. R.; BIGGAR, J. W. Geoestatistical theory and application to variability of some agronomical properties. Hilgardia, Berkeley, v. 51, n. 3, p. 1-75, 1983.

VIEIRA, S. R.; MILLETE, J.; TOPP, G. C.; REYNOLDS, W. D. Handbook for geostatistical analysis of variability in soil and meteorological paramaters. In: ALVAREZ V. H. Tópicos em ciências do solo II.Viçosa, MG: Sociedade Brasileira de Ciência do Solo, 2002. v. 2, p.1-45.

WACKERNAGEL, H. Multivariate geostatistics. $3^{\text {rd }}$ ed. Berlin: Springer,.2003. 